\title{
Ionel Bușe
}

\section{Johann Moritz}

\section{ou « l'homme sans identité »}

JOHANN MORITZ OR “THE MAN WITHOUT IDENTITY"

Abstract: My article concerns the analysis of the several "identities" of Johann Moritz (alias Anthony Quinn) in the film directed by Henri Verneuil, The Twenty-Fifth Hour (a French-Italian-Yugoslavian coproduction), produced by Carlo Ponti, and adapted after a book published by the exiled Romanian novelist Virgil Gheorghiu in Paris in 1949. Johann Moritz or the "man of Fontana" is the “man of La Mancha”, but unlike Cervantes's mad knight who wants to rid the world of monsters, he is a pacifist dreamer, embodying the drama of identity dissolution, under the pressure of the totalitarian regimes of the 2oth century.

Keywords: Virgil Gheorghiu; The Twenty-Fifth Hour; Johann Moritz; Identity; Heteronym; Exile; Totalitarian Regimes.

\section{IONEL BUȘE}

Centre « Mircea Eliade », Université de

Craiova, Roumanie

ionelbuse@yahoo.com

DOI: 10.24193/cechinox.2021.41.25
T a question de l'identité commence Là être posée d'une manière différente au début du $\mathrm{XX}^{\mathrm{e}}$ siècle par rapport au $\mathrm{XIX}^{\mathrm{e}}$ siècle après la déconstruction du sujet romantique et l'apparition de la psychanalyse et des avant-gardes. Une nouvelle perspective est annoncée par les poètes et les romanciers de l'époque. Pour l'écrivain portugais Fernando Pessoa, par exemple, l'identité du sujet est une identité hétéronyme ; il construit plusieurs hétéronymes par le dédoublement de sa personne ; il vit plusieurs vies (ses exégètes ont trouvé soixante-douze en 1990). Nous mentionnons les trois principaux noms qu'il emploie (Alberto Caeiro, Alvaro de Campos et Ricardo Reis) et qui entrent souvent en conflit avec lorthonyme Fernando Pessoa. Le jeu de sa construction poétique offre une totale liberté aux trois personnages : " $\hat{A} m e$ errante, selon ses propres termes, c'est par l'écriture des autres lui-même que Pessoa a voyagé de personne en personne, vivant de multiples vies par le biais de la construction de ce spectacle en lui et hors de lui ${ }^{1}$.

Reprenant l'idée d'hétéronomie de Fernando Pessoa nous analyserons l'identité multiple de Johann Moritz (alias Anthony Quinn) du film «La 
Vingt-cinquième heure », (une coproduction France, Italie, Yougoslavie), mis en scène par Henri Verneuil, et produit par Carlo Ponti ; ce film est réalisé d'après le livre La vingt-cinquième heure du romancier roumain Virgil Gheorghiu, paru en 1949 à Paris. Si Fernando Pessoa construit lui-même ses hétéronymes, l'hétéronomie identitaire de Johann Moritz est imposée par la «terreur de l'histoire $»^{2}$. L'expression "l'homme sans identité » est la paraphrase du titre du roman inachevé de Robert Musil, "L'homme sans qualités ». Même si l'écrivain Virgil Gheorghiu n'appartient pas au même courant littéraire, le modernisme viennois, les deux romans partagent la même idée générale de la crise de la modernité : l'aliénation individuelle et collective due à l'emprise de la machine idéologique du $\mathrm{XX}^{\mathrm{e}}$ siècle sur les hommes.

\section{L'homme de Fontana}

$\mathrm{L}$ e film La vingt-cinquième heure nous emmène en Transylvanie, dans un village roumain nommé Fontana (La Fontaine, en roumain "Fântâna »), durant l'année fatidique 1939, le 15 mars (le jour de l'invasion de la Tchécoslovaquie). Dans cette communauté, située en dehors du temps, les gens soccupent de leur traintrain quotidien, c'est-à-dire des travaux de la terre, de l'élevage des animaux, etc. ; parfois, lors des événements, tel le mariage, le baptême, l'enterrement, etc., ils sortent de leur routine. Ce n'est pas un hasard si le film commence par le baptême du deuxième fils de la jeune famille paysanne Moritz. Leurs noms et prénoms, Johann et Suzanna Moritz, sont semi-germanisés ; ils font probablement référence à l'époque d'avant la première guerre mondiale quand la Transylvanie faisait partie de l'Empire Austro-Hongrois. Le prénom roumain de Johann Moritz est Ioan. Le nom, qui est d'origine allemande, est aussi le résultat d'une germanisation. On retrouve le même nom dans certaines communautés juives de l'espace allemand.

À la célébration du baptême orthodoxe du petit Moritz participe tout le village. Les paysans portent tous des costumes traditionnels. Ils jouent et dansent comme dans les sociétés rurales de l'Europe Centrale et de l'Est. Sans doute, le baptême a des multiples significations ; en outre, il acquiert des significations supplémentaires par le biais du scénario. L'enfant vient au monde, tout comme dans les contes de fées, sous un mauvais destin. La technique, qui lui apporte de la tranquillité par la musique douce d'un appareil de radio, se transforme dans un monstre infatigable. En pleine fête l'enfant se met à pleurer de toutes ses larmes. Ses proches se demandent ce qui se passe. D'un coup, sur la même station radio, la pièce musicale a été remplacée par une voix forte et menaçante. «La voix réveillerait un cheval mort » constate Johann Moritz. C'est la voix d'Hitler. "Les allemands ont envahi la Tchécoslovaquie ", dit le jeune écrivain Trajan Coruga, le fils du pope du village, qui vient d'arriver de Bucarest à Fontana, pour présenter sa fiancée à ses parents. Si le paysan Johann Moritz semble être l'homme le plus innocent du monde, l'écrivain Traian Coruga, son ami d'enfance, représente la voix intellectuelle la plus consciente et tragique du film. Mais c'est une voix qui se fait entendre par Johann Moritz. Les villageois ont un grand respect pour leur pope Coruga et pour son fils, l'intellectuel Trajan (l'alter ego du romancier Virgil Gheorghiu). 
D'ailleurs le roman « La vingt-cinquième heure " se fonde sur une partie de la biographie de l'auteur.

Trajan considère qu'à cette époque-là on ne pouvait plus écrire des fictions. Mais que peut-on écrire ? La réponse de Trajan est dramatique : "C'est la vingt-cinquième heure ». Toutes les 24 heures ont été épuisées - le monstre est déjà réveillé. "Qui survivra parmi nous ? » se demande-t-il en regardant par la fenêtre la danse frénétique des paysans.

Un aspect intéressant du début du film est le rêve américain des jeunes de Fontana. Depuis la fin du XIX ${ }^{\mathrm{e}}$ siècle, l'Amérique du Nord est devenue l'El Dorado des gens de l'Europe de l'Est. Johann Moritz avouait au parrain de son deuxième fils, qui s'apprêtait à embarquer le lendemain le navire "Danube " pour partir en Amérique, qu'il a eu lui aussi ce rêve avant de se marier. Comme tous les jeunes paysans de Transylvanie, il voulait gagner de l'argent et retourner à Fontana pour acheter de la terre et construire une maison ${ }^{3}$. Mais l'amour pour sa fiancée Suzanna l'a fait rester à Fontana. Trois symboles identitaires, la terre, le village de Fontana et sa femme Suzanna, accompagnent Johann Moritz dans tous les camps de travaux où il sera déporté.

\section{Le juif errant}

Ud n simple fonctionnaire peut changer définitivement le destin et l'identité d'un homme. C'est le cas du sergent major Nicolae Dobrescu de Fontana. Pour avoir la belle Suzanna comme maîtresse, même si elle le rejette chaque jour, ce dictateur local inscrit son époux, Johann Moritz, sur la liste des juifs qui devraient être déportés dans un camp de travail. Nous sommes en 1940, dans un régime de dictature, où les lois antisémites étaient appliquées de manière expéditive. Johann Moritz et le marchand juif Marco Golberg de Fontana sont escortés vers un camp de travail. Johann Moritz essaie de convaincre Marco de témoigner que lui, Johann n'est pas d'origine juive. Il sait que la déportation est une injustice envers les Juifs, mais pour lui, qui est Roumain, c'est une erreur. Marco Goldberg refuse de témoigner : "Quelle est la différence entre l'erreur et l'injustice aujourd'hui ? Nous vivons dans un monde où n'importe qui peut devenir juif », réplique-t-il. Toutes les démarches de Johann Moritz auprès du commandant du camp pour réparer l'erreur échouent. Ses lettres envoyées à Suzanna sont lues et jetées à la poubelle par celui-ci. Cependant Johann Moritz vit toujours avec l'espérance que cette grande erreur sera un jour corrigée.

Nous entrons dans un scenario kafkaïen. D’un côté, les Juifs lui reprochent qu'il ne veut pas parler yiddish et reconnaître ainsi qu'il est juif, d'autre côté, les autorités ne veulent pas chercher la vérité. « Le roi n'a jamais tort » dit un fonctionnaire. Les démarches faites par sa femme Suzanna, par le pope Coruga et son fils Trajan auprès du Ministère de la Force Armée à Bucarest sont inutiles. Johann Moritz est transformé finalement en une simple annexe d'un système autocratique qui fonctionne comme une machine à vide. Mais paradoxalement l'homme de Fontana ne perd pas ses qualités humaines. Il rêve qu'un jour, après la guerre, il amènera sa femme et ses fils visiter le grand canal qu'il a construit.

Entre-temps l'armée allemande envahit la Roumanie ; la situation devient plus 
compliquée. Suzana est obligée de divorcer afin de ne pas se faire confisquer ses biens, son mari étant considéré Juif. En recevant la lettre de divorce, Johann refuse de croire que Suzanne veut divorcer : «Elle n'est pas comme les autres femmes ", explique-t-il au commandant. Pour les autorités ce fait prouvait qu'il est Juif. Finalement, Johann Moritz commence à penser à son tour qu'il est Juif: « Peut-être que mon grand-père ou quelqu'un était Juif », dit-il en se confessant au commandant du camp. Et il s'intègre petit à petit à la communauté des Juifs en apprenant le yiddish. Ses camarades l'appellent Iankel. Un jour, quelques détenus se préparent pour évader du camp. Ils tentent de convaincre Johann de se joindre à eux. Ils avaient besoin de lui pour leur porter leurs lourds bagages. Surprise! En pleine nuit la voiture du commandant conduite par luimême quitte le camp avec le petit groupe et part vers la frontière avec la Hongrie où, à l'époque, fonctionnait un Comité d'Aide pour les Juifs qui organisait le transport de leurs compatriotes en Amérique. Même si Johann Moritz ne comprend pas grandchose, quand il entend le mot «Amérique " son ancien rêve lui revient. Mais cette fois il semble qu'il n'est pas trop enthousiaste. "Amérique...Toujours Amérique... ", dit-il avec une voix sourde.

\section{Le hongrois " volontaire "}

$A_{\text {est logé clandestinement dans la }}^{\text {rrivé à Budapest, le groupe des Juifs }}$ maison de leurs compatriotes jusqu'au départ, le jour suivant. Johann Moritz découvre que les Roumains sont indésirables en Hongrie. Il est logé dans un coin de la cuisine où s'occupe de lui une jeune femme qui fait la cuisine et le ménage. Elle s'appelle Iuliska et Johann parle avec elle en hongrois. Comme il venait d'une région multiethnique de l'ancien Empire et il parlait l'allemand et le hongrois. Iuliska l'invite à manger. «Je suis Roumain ", l'avertit Johann en masquant son identité. "Et les Roumains ne mangent pas ? " répond-elle en souriant. La simplicité d'Iuliska est humaine. Les deux se situent au-delà des nationalismes et des idéologies du siècle.

Le jour suivant, il est annoncé par son compagnon juif que le Comité d'Aide pour les Juifs ne peut pas lui donner le document pour partir en Amérique parce qu'il n'est pas Juif. Johann lui demande comment il est possible d'être Juif pendant un an et demi et puis changer de nationalité. La réponse de son compagnon est implacable : "Devant les balles, vous pouvez être Juif mais pas parmi les Juifs! ». Parmi les Juifs il a perdu cette qualité humaine. "Peut-être qu'il y a aussi un Comité pour aider les chrétiens ? ", demande naïvement Iohann Moritz. Malheureusement les chrétiens nont pas eu un Comité pour sauver les chrétiens. Dans la question posée par Johann Moritz, on découvre toute l'impuissance tragique de l'Europe chrétienne devant le mal.

Pour compenser l'aide reçue pendant leur voyage son compagnon juif lui offre un objet d'or. «L'humanité » de Johann Moritz a un prix comme toute autre marchandise. Parce qu'il n'avait pas de papiers, la police hongroise le met en prison. Les soupçons de la police augmentent lorsqu'ils découvrent l'objet d'or russe (c'est-à-dire soviétique !). De plus, lorsque Johann Moritz avoue être roumain, il est battu et torturé parce que les Hongrois l'avaient considéré espion. À l'époque entre la Roumanie et la Hongrie existait un conflit politique majeur : le 
Nord-Ouest de la Transylvanie a été attribué à la Hongrie par le Diktat de Vienne du 30 août 1940, arbitré par Hitler et Mussolini. Cela avait comme but la participation de la Hongrie à la guerre d'alliance entre les puissances de l'Axe. Pour montrer sa loyauté à Hitler, Horthy, le régent du royaume de Hongrie, s'engage à envoyer plusieurs milliers de Hongrois en Allemagne pour travailler dans l'industrie allemande. Mais ces travailleurs ne sont pas des Hongrois; ils appartiennent à d'autres nationalités indésirables en Hongrie (Croates, Polonais, Tchèques, Français, Roumains etc.). La Police hongroise change donc le nom de Johann Moritz en Ianos Moritz et l'embarque dans le train de "l'amitié " hongro-allemande qui part vers le camp de travail d'Orenbourg - Allemagne. Ces faux Hongrois ne représentent que des chiffres sans identités. Avant de passer la frontière ils sont obligés par les soldats hongrois de cueillir des fleurs pour décorer le train. Au camp d'Orenbourg les faux volontaires sont annexés à la machine allemande de la guerre. À côté de chaque ouvrier se trouve un soldat allemand qui supervisait son travail. L'Allemand menace Ianos Moritz de mort s'il couche avec des femmes allemandes. C'est un sabotage. "Le hongrois " Ianos Moritz est moqué aussi par son gardien allemand qui le prend pour un barbare mongol. Johann proteste : il avoue être Roumain. Le soldat nazi lui répond que pour lui c'était la même chose : Hongrois, Roumain, Mongol. Ce ne sont pas de vraies identités.

\section{Le membre de la famille héroïque}

$\mathrm{E}$ ntre temps une équipe de médecins qui visitaient les camps de travail s'arrête devant Johann Moritz. Le colonel, médecin chef, s'excite soudainement. Une grande surprise identitaire se produit. L'absurde se met en place dans l'histoire linéaire de l'humanité. L'homme de Fontana, Johann Moritz, est declaré l'homo universalis, l'homme de la race pure. Lui qui n'avait plus d'identité parce que l'histoire lui avait volé à chaque fois, devient lêtre humain le plus connu par les gens. La science médicale crée l'histoire (tout comme aujourd'hui !). La science et la technologie se mettent au travail. Les traits du visage de Johann Moritz sont attentivement mesurés par le médecin de Goebbels et de Rosenberg. Il fait sa démonstration devant les jeunes médecins militaires. Centimètre par centimètre, la forme de la tête, les os du visage, etc. sont mesurés avec le compas. Ecce homo! Un membre de la race pure germanique ! «Un membre de la famille héroïque ", conclut le colonel. "Connaissez-vous ma famille, colonel ?" demande naïvement Johann Moritz. « Mieux que toi, mon fils ", lui répond le colonel.

D'un côté il y a l'humain et d'autre côté la science de l'humain transformée en utopie politique. L'homme nouveau est le plus ancien et le plus pur, grâce à la science du national-socialisme de la race aryenne. «L'aryen » de l'Ouest et « le prolétaire » de l'Est sont deux bons amis; ceux-ci avaient prévu de partager le monde « scientifiquement » sous les regards impassibles d'autres somnambules de l'histoire.

Johann Moritz devient le plus célèbre soldat SS. Il est mis à surveiller son ancien camp de travail. L'un de ses amis, un français, qui l'appelait Jean, lui demande d'où il s'est procuré l'uniforme en croyant qu'il se prépare pour évader. «Non, je suis sentinelle, répond Johann. Le colonel connaît 
ma famille ». La situation comique tend à atteindre l'absurde. Le colonel montre à Johann sa photo sur la couverture d'une revue nazie. Johann en est ravi. Stupidité ? Simple naïveté ? C'était probablement la première fois qu'il se voyait sur une photographie.

Vers la fin de la guerre l'aviation américaine attaque le camp. Les soldats allemands et beaucoup de prisonniers sont tués. Mais les prisonniers sont très enthousiastes. "Américains ou Anglais ? " demandent-ils joyeusement. " Ça m’est égal. Pour l'instant nous sommes ici sous leurs bombes » répond Johann. Puis il aide à évader les prisonniers de son escorte en tuant des soldats allemands. C'est le seul épisode où il a utilisé la force pour protéger les prisonniers sans défense. Arrivé sur le territoire américain, même s'il a aidé ses anciens compatriotes du camp de travail, il est mis en prison à cause de sa "participation » à la guerre comme soldat SS. Sa photographie était sur les couvertures de toutes les revues nazies. Le même camp d'Orenbourg devient un camp américain pour les prisonniers de la guerre.

Dans le camp d'Orenbourg Johann rencontre son ami de Fontana, monsieur Trajan Coruga qui, après avoir été prisonnier des Allemands en tant qu'intellectuel roumain indésirable, est devenu prisonnier des Américains parce qu'il était Roumain : les Roumains avaient été les alliés des Allemands pendant la première partie de la guerre. Durant les dix-neuf mois passés dans le camp, Trajan Coruga écrit soixante-quatre lettres adressées au commandant pour lui expliquer la situation bizarre dans laquelle se trouvaient les deux (Trajan Coruga et Johann Moritz) qui n'avaient pas été des combattants de guerre ; il ne reçoit aucune réponse. Les prisonniers sont traités comme de simples chiffres. Johann Moritz est appelé chez le commandant pour une question qui visait son nom : s'écrit-il avec $t z$ ou avec $z$ ? Le commandant n'a pas le temps d'écouter son histoire.

Entre temps Trajan Coruga reçoit une lettre de Fontana ; il apprend que sa mère est morte et son père a été emprisonné par les communistes qui ont fermé l'église. Il avait également perdu sa femme déportée par les Allemands parce quelle était Juive. Trajan raconte à Johann que sa famille est vivante; elle s'est cachée à cause des communistes. La photo de Johann était partout dans le territoire occupé par l'Allemagne. Johann se rend compte que Trajan veut se suicider et essaie de l'arrêter : "Il y a toujours quelque chose de beau à regarder ». Johann lui rappelle qu'il doit écrire le livre promis, "La vingt-cinquième heure ». "C'est la dernière heure ", tout est fini. Johann ne comprend pas. Le matin, Trajan se rend pour protester à la clôture de barbelés du camp, la soixante-cinquième lettre adressée au commandant à la main. Après quelques avertissements de la sentinelle, il reçoit une balle dans la tête.

Johann Moritz est accusé de crimes contre l'humanité devant le tribunal de Nuremberg. Des millions de personnes sont mortes à cause de lui selon l'avocat de l'accusation qui exige que les coupables soient condamnés à mort. Lavocat de la défense pose une seule question à Johann : "Moritz, tu sais pourquoi tu es dans cette salle d'audience? " "Monsieur, au cours des huit dernières années, je n'avais aucune idée pourquoi jétais à certains endroits". L'avocat lit ensuite devant le tribunal une lettre dramatique adressée de Suzanna à 
Johann. L'avocat de la défense demande de la pitié pour Johann Moritz.

\section{L'exil de l'humain}

L 'homme de la Fontana est "l'homme de la Mancha ", mais contrairement au chevalier-fou de Cervantès qui veut libérer le monde des monstres, il est un rêveur pacifiste qui incarne le drame de l'humain. À Johann Moritz lui manque partout son village Fontana et il garde toujours son humanité. Il humanise son travail. Il est heureux que les détenus aient fini le canal. Il se réjouit quand il voyage pour la première fois en train, même si le train va dans un camp de travail allemand. Il est fier de sa photo en uniforme nazi sur la revue «Signal ». C'était pour la première fois qu'il voyait une photo de lui. Il croit que tout est dû à l'officier qui connaissait sa famille. En même temps il aide les prisonniers à s'évader en fusillant les gardiens allemands.

Trois ans après le procès de Nuremberg, en 1949, Johann Moritz est libéré. Mais il est obligé de rester en exil. Les soviétiques avaient envahi la Roumanie. Le nouveau temps est un temps hybride, sans identité. Si dans les camps de travaux forcés il pourrait au moins rêver à son retour à Fontana (comme Ulysse), c'était parce que Fontana était encore là, sa femme et ses fils aussi. Maintenant Suzanne est sur le quai de la gare d'une petite ville allemande. Elle n'est pas la même. Violée par les soldats soviétiques, tout comme la terre de Fontana, elle devient une autre femme. Le fils né du « viol de l'histoire » est un être hybride. Toute la famille est maintenant hybride. Lexil devient une fausse patrie.

Dans la dernière image du film, le reporter photographe, qui voulait prendre une photo des membres de la famille
Moritz sur le quai de la gare, essaie de surprendre la joie de leur libération et de leurs retrouvailles. C'était pour le marketing de son journal américain. Il a une attitude imposante parce que la famille des paysans ne connaît pas les règles des nouveaux Médias. La photo est une image réifiée, l'image cynique du nouveau monde. Le petit enfant est placé dans les bras de Johann Moritz ; ce dernier est obligé de sourire. Il accepte, bien sûr, c'est un geste humain et paternel, mais il ne peut pas sourire. «Souris! Souris!», lui ordonne le reporter. Son sourire est triste. C'est la tristesse de l'humain en exil.

\section{En guise de conclusion}

T a tragédie des personnages du film $L a$ Luingt-cinquième heure " (Johann Moritz, Suzana Moritz, Trajan Coruga, etc.) fait part de la grande tragédie de l'homme du $\mathrm{XX}^{\mathrm{e}}$ siècle. Elle relève la décadence apocalyptique de l'Europe et le processus de deshumanisation de l'humain par sa soumission aux systèmes idéologiques de la barbarie moderne : le nazisme et le communisme.

Johann Moritz perd tous ses attributs humains individuels par l'aliénation absolue de son identité. L'hétéronomie de cette identité n'est pas un jeu mais une destruction lente des possibilités individuelles créatrices. L'homme aux identités multiples devient le citoyen sans identité. Dans un passage du livre est formulée l'essence de cette nouvelle apocalypse :

Le citoyen est un homme qui ne vit que la dimension sociale de la vie. C'est comme un piston de la machine, il ne fait qu'un seul mouvement et le répète à l'infini. Le citoyen est la bête 
la plus apocalyptique, apparaissant sur la face de la terre en croisant l'homme avec l'esclave technique. Il a la cruauté de l'animal et la froide indifférence de la machine. Le type de citoyen le plus parfait a été créé par les Russes : Le commissaire!
En même temps, il ne faut pas oublier que l'exil pour Johann Moritz est un nouvel hétéronyme de sa soumission. Il est devenu l'homme sans patrie dans un monde de plus en plus individualiste, pragmatique, hédoniste, imposé par les nouvelles idéologies de l'Occident américanisé.

\section{BibLIOGRAPHIE}

Gheorghiu, Constantin Virgil, Ora 25, Bucarest, Ed. Omegapres, 1991 ; La Vingt-cinquième Heure (roman), Paris, Plon, 1949.

Ferréol, Gilles et Guy Jucquois, Dictionnaire de l'altérité et des relations interculturelles, Paris, A. Colin, 2003.

Wunenburger, Jean-Jacques, Le Sacré, Paris, PUF, 2009 ; Une utopie de la raison : essai sur la politique moderne, Paris, La Table Ronde, 2002.

Cinemagia, 16. 04. 2016 : https://magazinweb.net/film-a-25-a-0ra-la-vingt-cinquieme-heure-1967. html (consulté le 19.09.2021)

https://www.cairn.info/revue-psychanalyse-2009-1-page-113.htm

https://www.literaturadeazi.ro/rubrici/recenzii/identitatea-orei-25-2530

\section{Notes}

1. Filomena Iooss, L'bétéronymie de Fernando Pessoa, Psychanalyse, 2009/1 (n 14), p. 113 à 128 in https://www.cairn.info/revue-psychanalyse-2009-1-page-113.htm, (consulté le 12.12. 2020).

2. Mircea Eliade considère le roman de Virgil Gheorghiu emblématiques pour la „terreur de l'histoire qu'endurent ses personnages". Voir Emilia Ciordas, Identitatea orei 25 (Identité de la $25^{\mathrm{c}}$ heure) in https://www.literaturadeazi.ro/rubrici/recenzii/identitatea-orei-25-2530 (consulté le 12.12.2020).

3. Aujourd'hui la même situation est à retrouver en Europe de l'Est. 